\title{
Effects of the grafting of lanthanum complexes on silica Surface on reactivity: Influence on ethylene, propylene and 1,3-butadiene homopolymerization
}

\author{
Iker Del Rosal*, Ahmed Yahia, Laurent Maron*
}

1. Université de Toulouse ; INSA, UPS ; LPCNO (IRSAMC) ; 135 avenue de Rangueil, F-31077 Toulouse, France

2. CNRS ; UMR 5215 (IRSAMC) ; F-31077 Toulouse, France

Supporting Information 


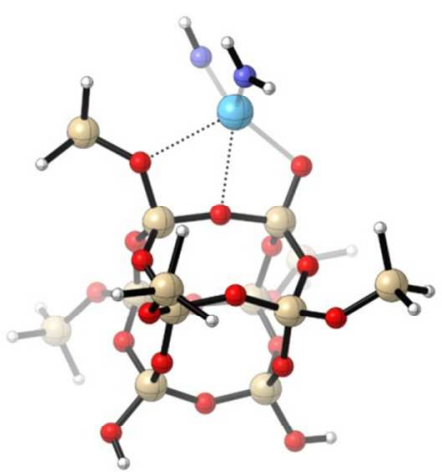

[Ln]@c-1

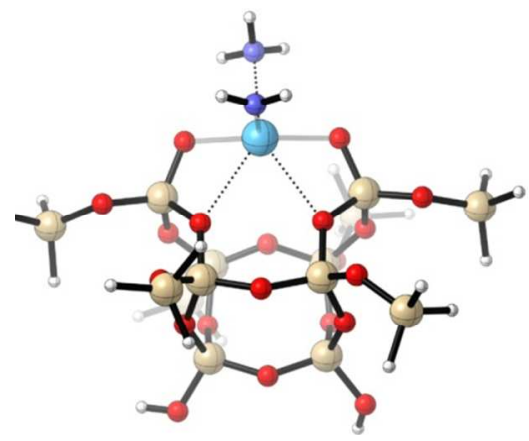

[Ln]@b-1

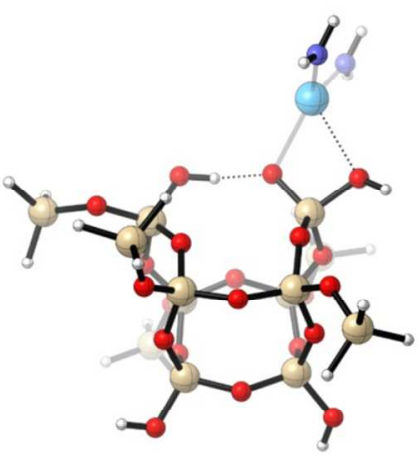

[Ln]@bc-3

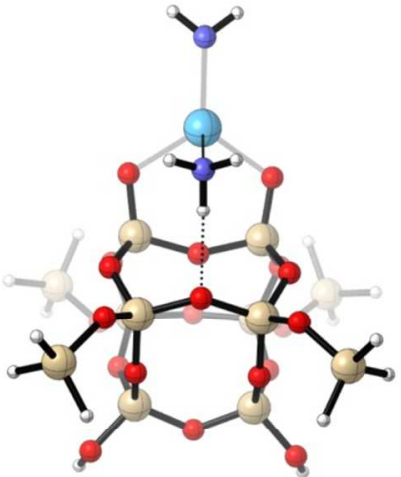

[Ln]@ac-2

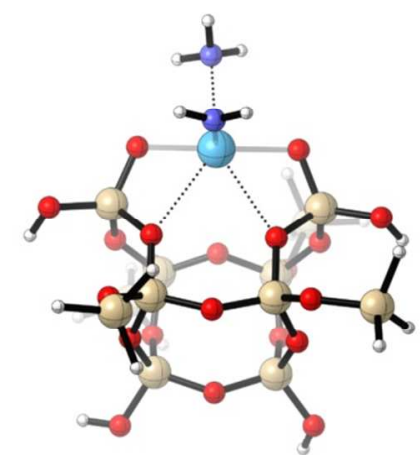

[Ln]@abc-1

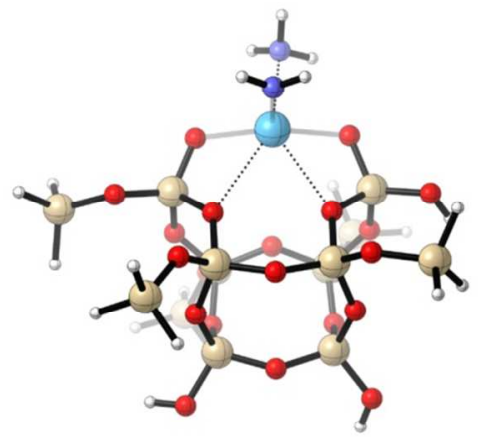

[Ln]@bc-1

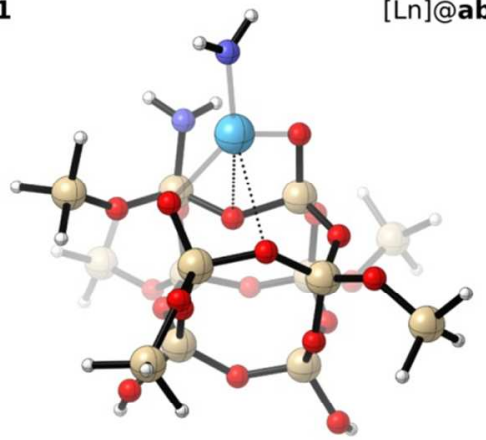

[Ln]@c-2

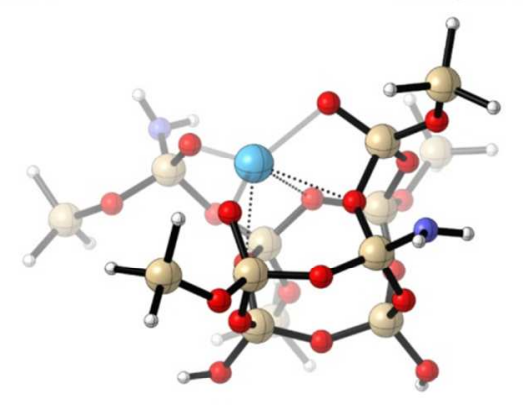

[Ln]@c-3

Figure S1. Representation of the grafting modes obtained by grafting a bis(timethyl)sylilamide lanthanide complex on the five systems, with different silanol groups, used as model of a silica surface dehydroxylated at $200^{\circ} \mathrm{C}$ 


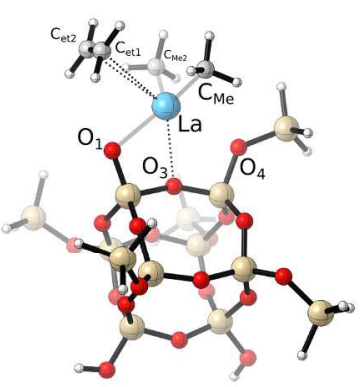

a) $A^{\text {et }}{ }_{c-1}$

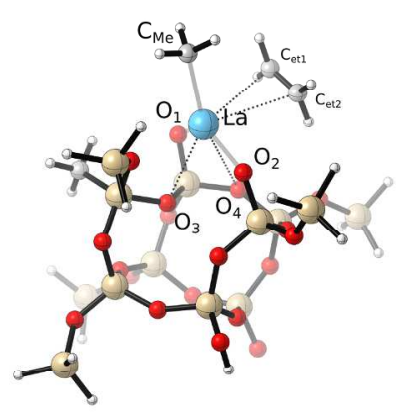

d) $A^{\text {et }}{ }_{c-2}$

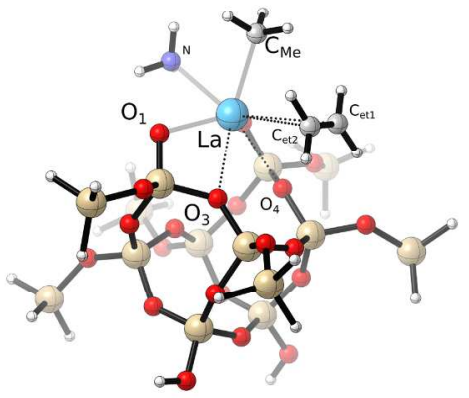

g) $A^{e t}{ }_{b-1}$

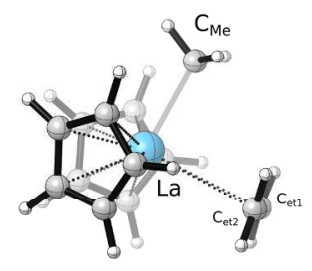

j) $A^{\text {et }} \mathrm{cp}$

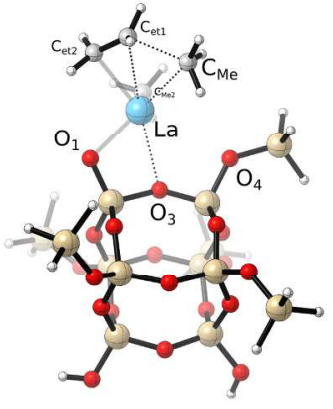

b) TS $\left(\mathrm{A}^{\mathrm{et}}{ }_{\mathrm{c}-1} \rightarrow \mathrm{B}^{\mathrm{et}}{ }_{\mathrm{c}-1}\right)$

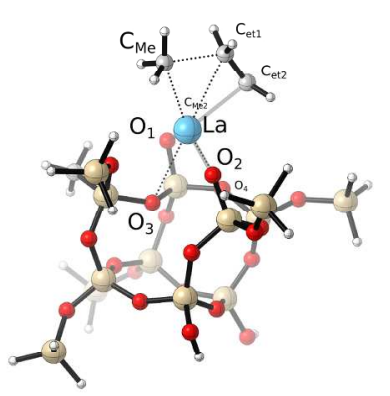

e) TS $\left(A^{e t}{ }_{c-2} \rightarrow B^{e t}{ }_{c-2}\right)$

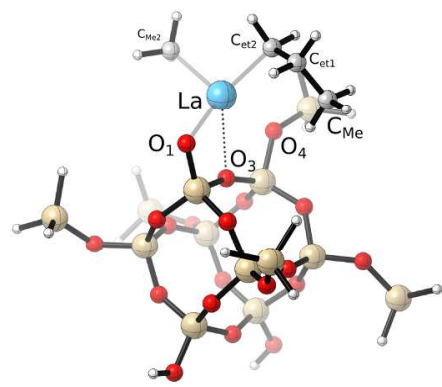

c) $B^{\text {et }}{ }_{c-1}$

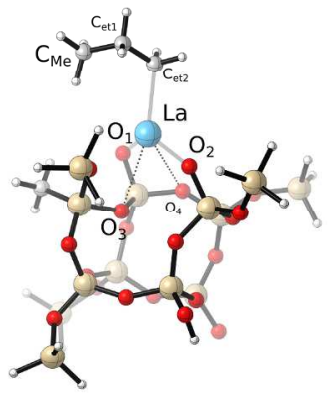

f) $B^{e t}{ }_{c-2}$

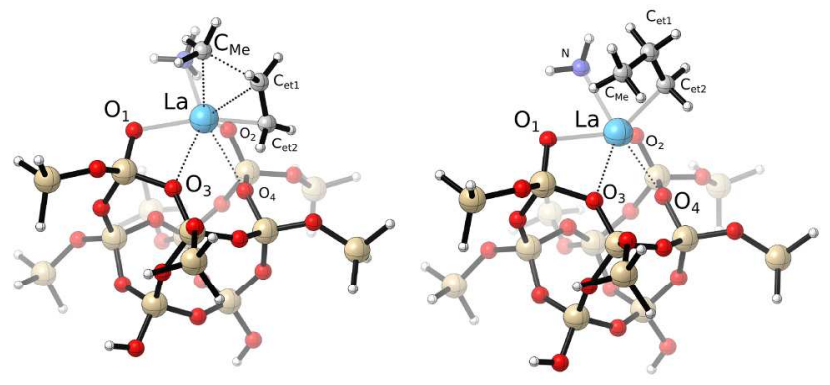

h) TS $\left(A^{e t}{ }_{b-1} \rightarrow B^{e t}{ }_{b-1}\right)$

i) $\mathrm{B}_{\mathrm{b}-1}^{\mathrm{et}}$

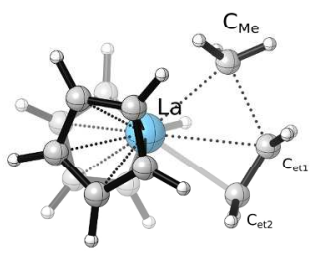

k) TS $\left(A^{\text {et }} C_{p} \rightarrow B^{\text {et }}{ }_{C_{p}}\right)$

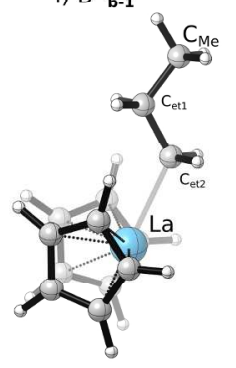

I) $B^{e t} c p_{p}$

Figure S2. Optimized structures of complexes involved in the initiation step of the ethylene polymerization mediated by [La]@c-1 ((a) to (c)), [La]@c-2 ((d) to (f)), [La]@b-1 ((g) to (i)) and $\mathrm{Cp}_{2} \mathrm{LaMe}\left((\mathrm{j})\right.$ to (1)). [La] refers to fragments $\mathrm{Si}_{14} \mathrm{O}_{21} \mathrm{H}_{20} \mathrm{La}(\mathrm{NH} 3)$ for [La]@b-1, and $\mathrm{Si}_{13} \mathrm{O}_{20} \mathrm{H}_{17} \mathrm{La}$ for [La]@c-1, and [La]@c-2 

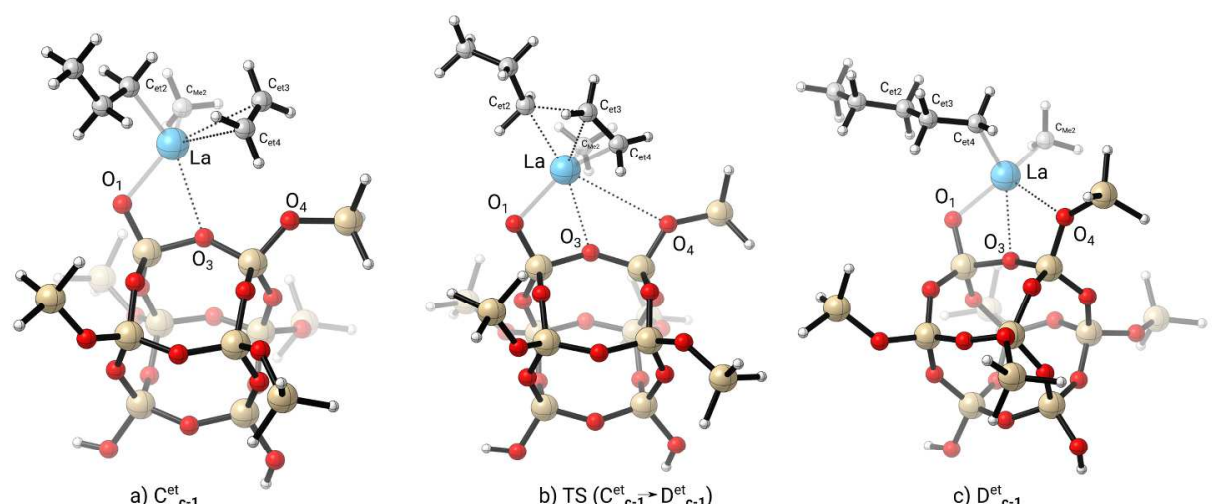

a) $\mathrm{C}_{\mathrm{c}-1}^{\mathrm{et}}$

$$
\text { b) } T S\left(C_{c-1}^{e t} D_{c-1}^{e t}\right)
$$

c) $D_{c-1}^{e t}$
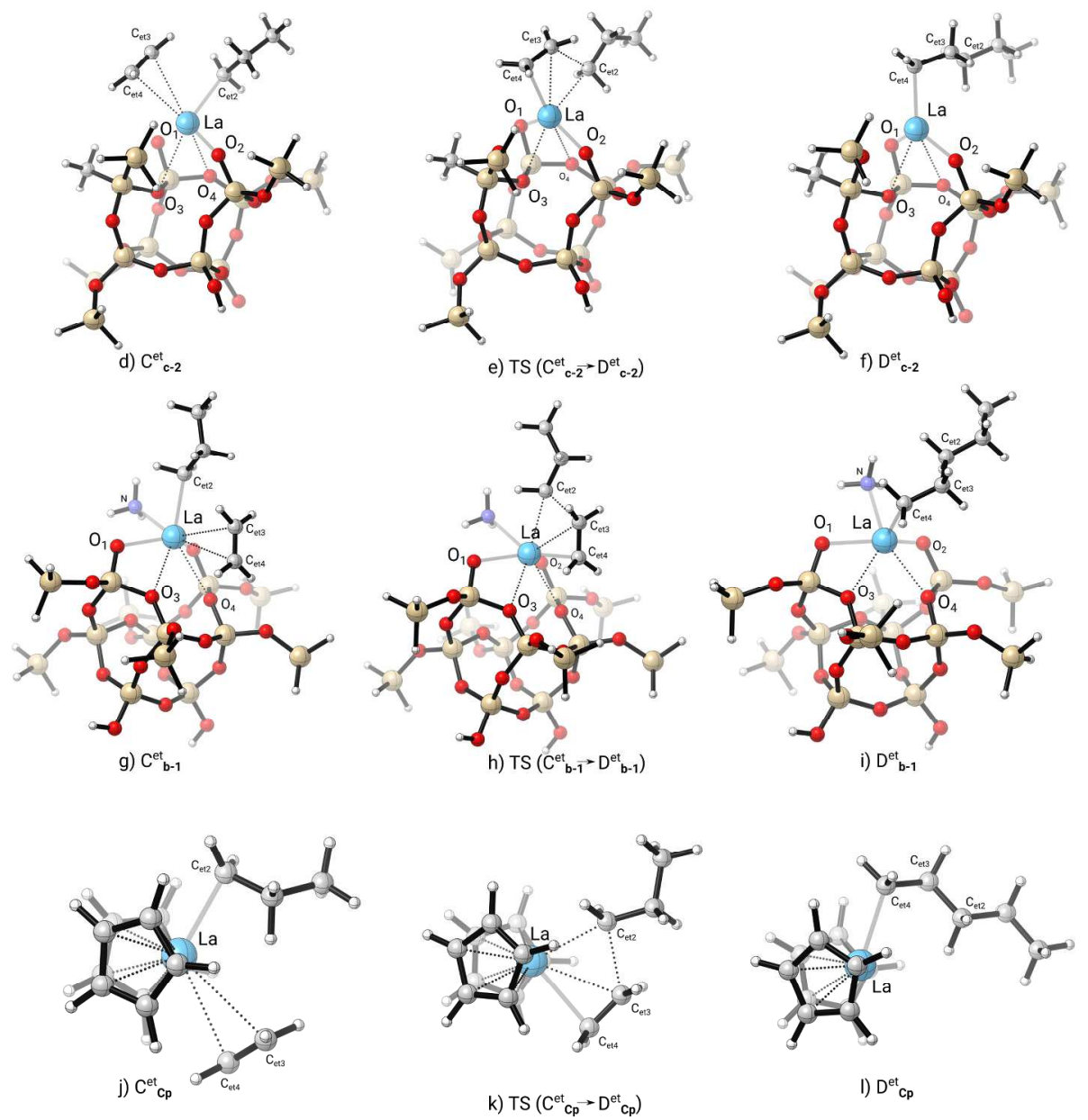

Figure S3. Optimized structures of complexes involved in the first propagation step of the ethylene polymerization mediated by by [La]@c-1 ((a) to (c)), [La]@c-2 ((d) to (f)), [La]@b-1 ((g) to (i)) and $\mathrm{Cp}_{2} \mathrm{LaMe}\left((\mathrm{j})\right.$ to (1)). [La] refers to fragments $\mathrm{Si}_{14} \mathrm{O}_{21} \mathrm{H}_{20} \mathrm{La}(\mathrm{NH} 3)$ for [La]@b-1, and $\mathrm{Si}_{13} \mathrm{O}_{20} \mathrm{H}_{17} \mathrm{La}$ for [La]@c-1, and [La]@c-2 


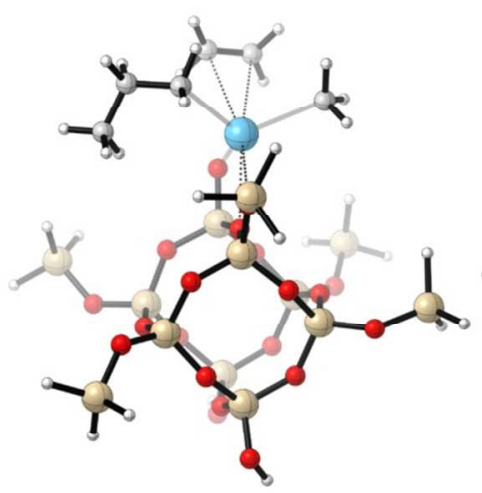

$A^{\prime e t}{ }_{c-1}$

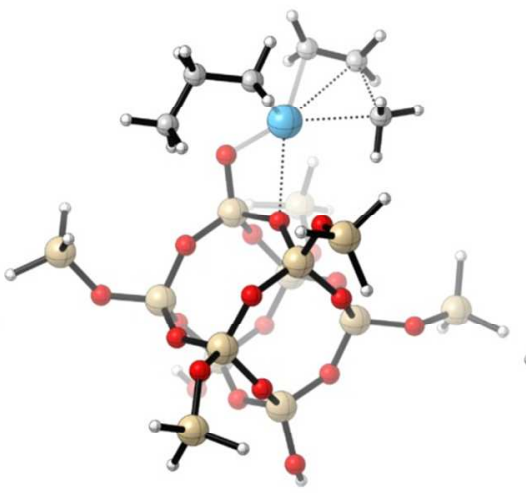

TS $\left(A^{\prime e t}{ }_{c-1} \rightarrow B^{\prime e t}{ }_{c-1}\right)$

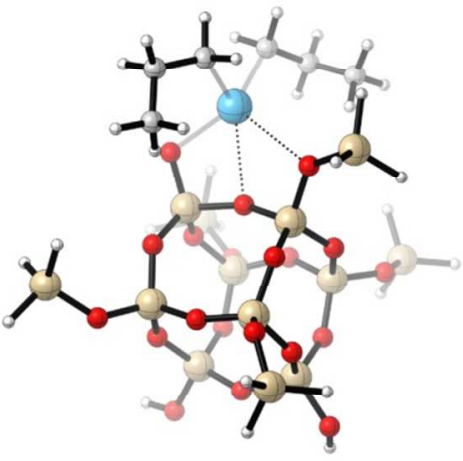

$B^{\prime \prime e t}$

Figure S4. Optimized structures of complexes involved in the insertion of an incomming ethylene monomer on the second [La] - Me arm of [La]@c-1. 

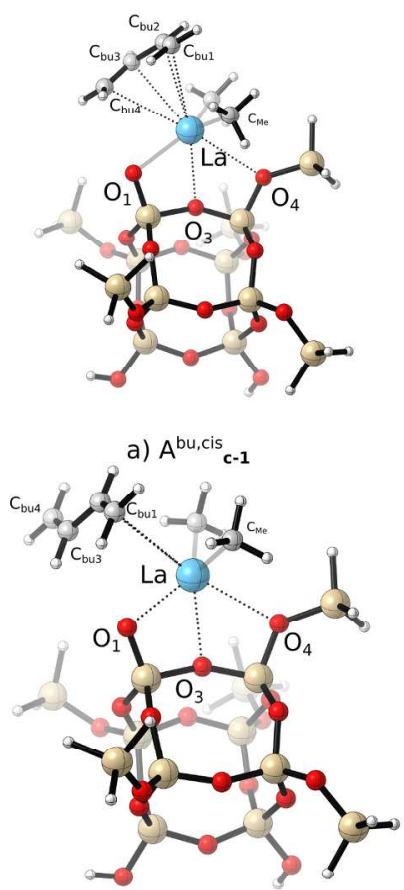

d) $A^{\text {bu,trans }}{ }_{c-1}$

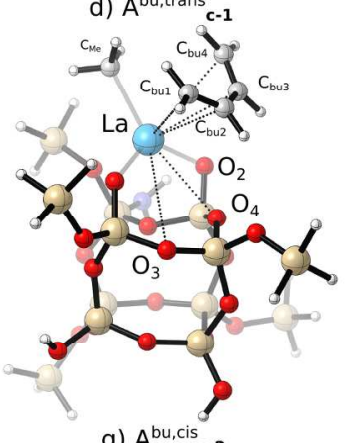

g) $A^{\text {bu,cis }}$ c-2

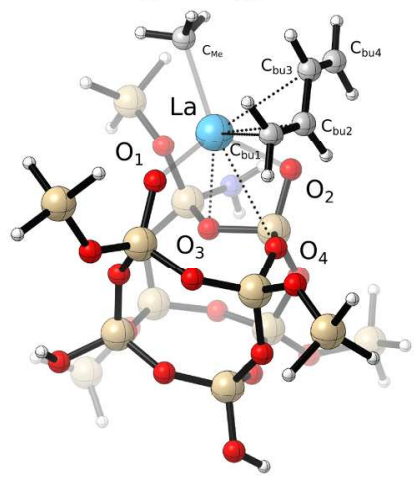

j) $A^{\text {bu,trans }}$ c-2

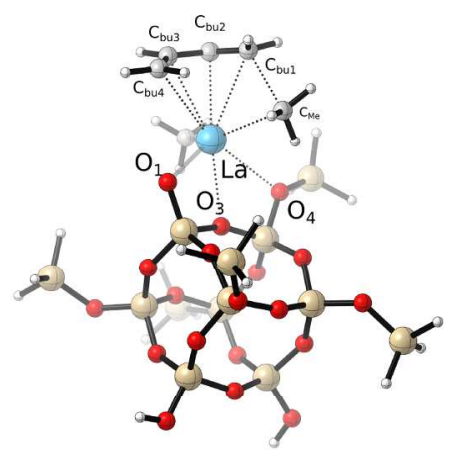

b) TS $\left(A^{\text {bu, is }}=-1 \rightarrow B^{\text {bu, is }} c_{c-1}\right)$
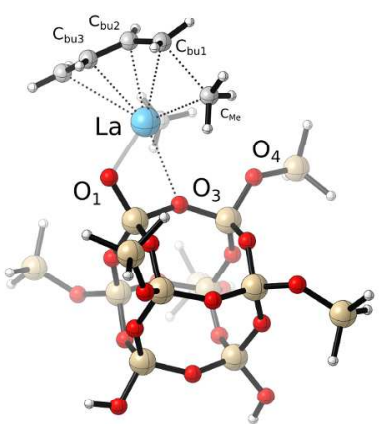

e) TS $\left(A^{\text {bu,trans }}{ }_{c-1} \rightarrow B^{\text {bu,trans }}{ }_{c-1}\right)$
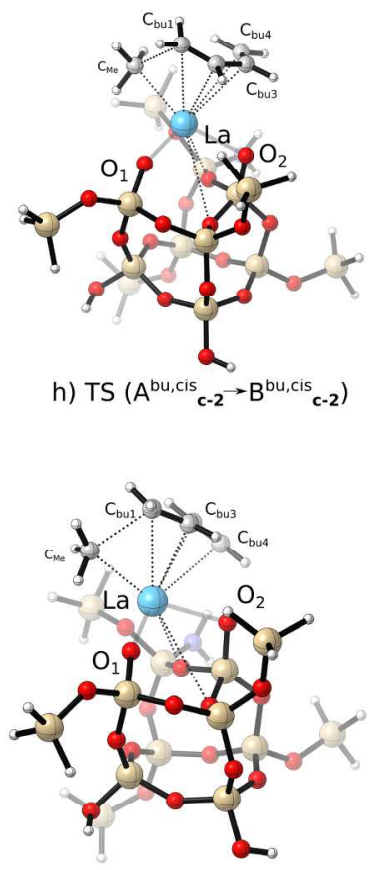

k) TS ( $\left.{ }^{\text {bu,trans }}{ }_{c-2} \rightarrow B^{\text {bu,trans }}{ }_{c-2}\right)$

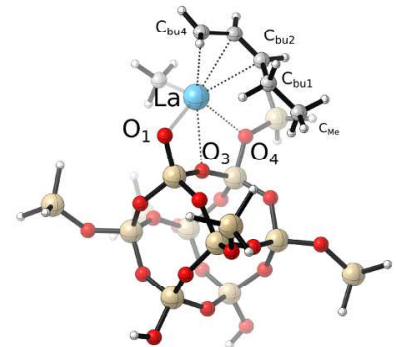

c) $\mathrm{B}^{\text {bu,cis }} \mathrm{c-1}$

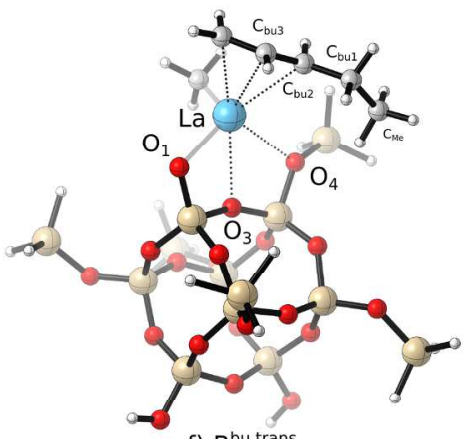

f) $B^{\text {bu, trans }}{ }_{c-1}$

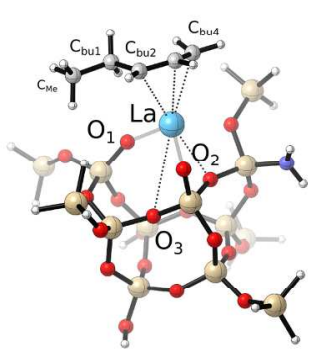

i) $\mathrm{B}_{\mathrm{c}-2}^{\mathrm{bu}, \mathrm{c}}$

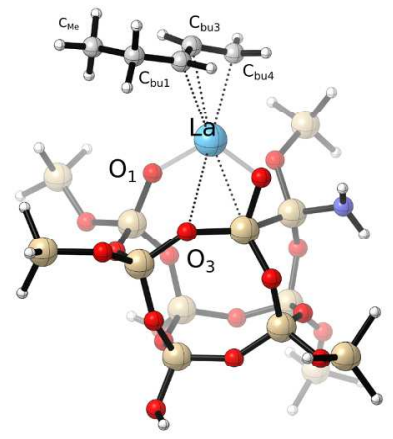

I) $B_{c-2}^{\text {bu,trans }}$

Figure S5. Optimized structures of complexes involved in the initiation step of the 1,3-butadiene polymerization mediated by [La]@c-1 ((a) to (f)) and [La]@c-2 ((g) to (1)). [La] refers to fragments $\mathrm{Si}_{13} \mathrm{O}_{20} \mathrm{H}_{17} \mathrm{La}$. 

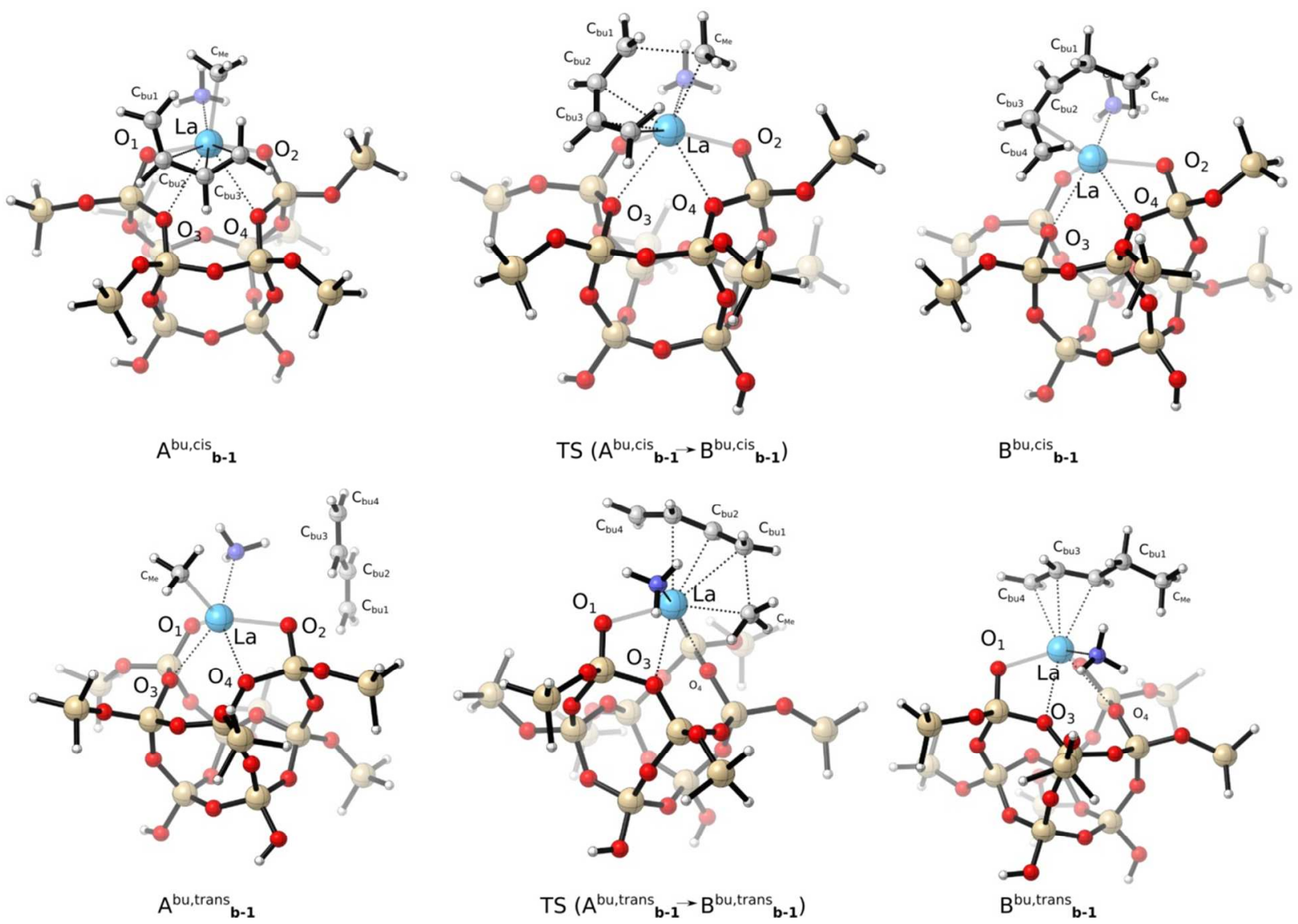

Figure S6. Optimized structures of complexes involved in the initiation step of the 1,3-butadiene polymerization mediated by [La]@b-1. [La] refers to fragments $\mathrm{Si}_{14} \mathrm{O}_{21} \mathrm{H}_{20} \mathrm{La}(\mathrm{NH} 3)$. 

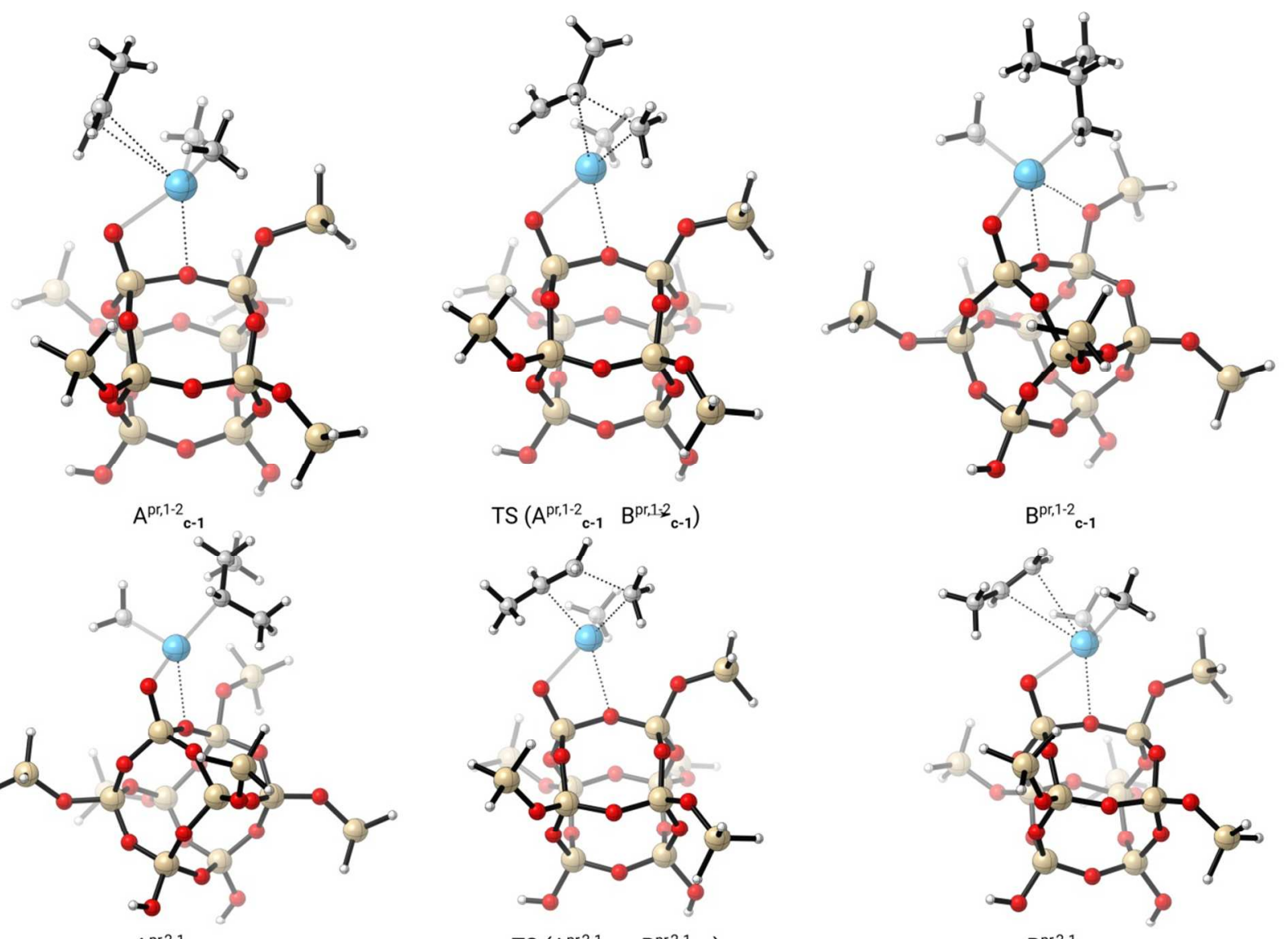

$\operatorname{TS}\left(A^{\mathrm{pr}, 2-1}{ }_{c-1} B^{\mathrm{pr}, 2 \mathrm{~d}}{ }_{c-1}\right)$

$\mathrm{B}^{\mathrm{pr}, 2-1}{ }_{\mathrm{c}-1}$

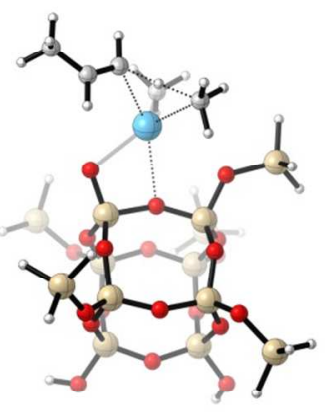

$\operatorname{TS}\left(A_{c-1}^{\text {privic }} B^{\text {pr,vis }}{ }_{c-1}\right)$

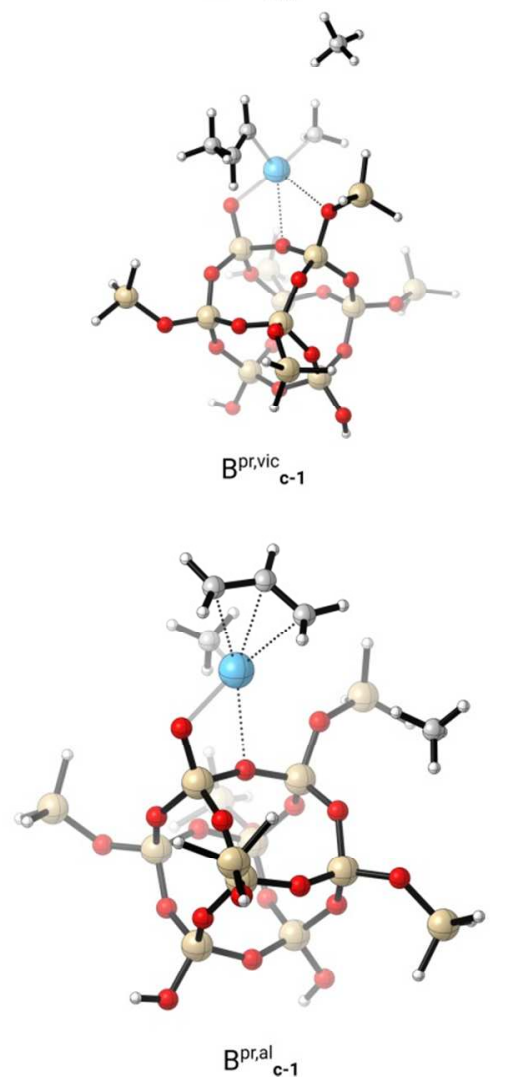


Figure S7. Optimized structures of complexes involved in the initiation step of the propylene polymerization mediated by [La]@c-1. [La] refers to fragments $\mathrm{Si}_{13} \mathrm{O}_{20} \mathrm{H}_{17} \mathrm{La}$.
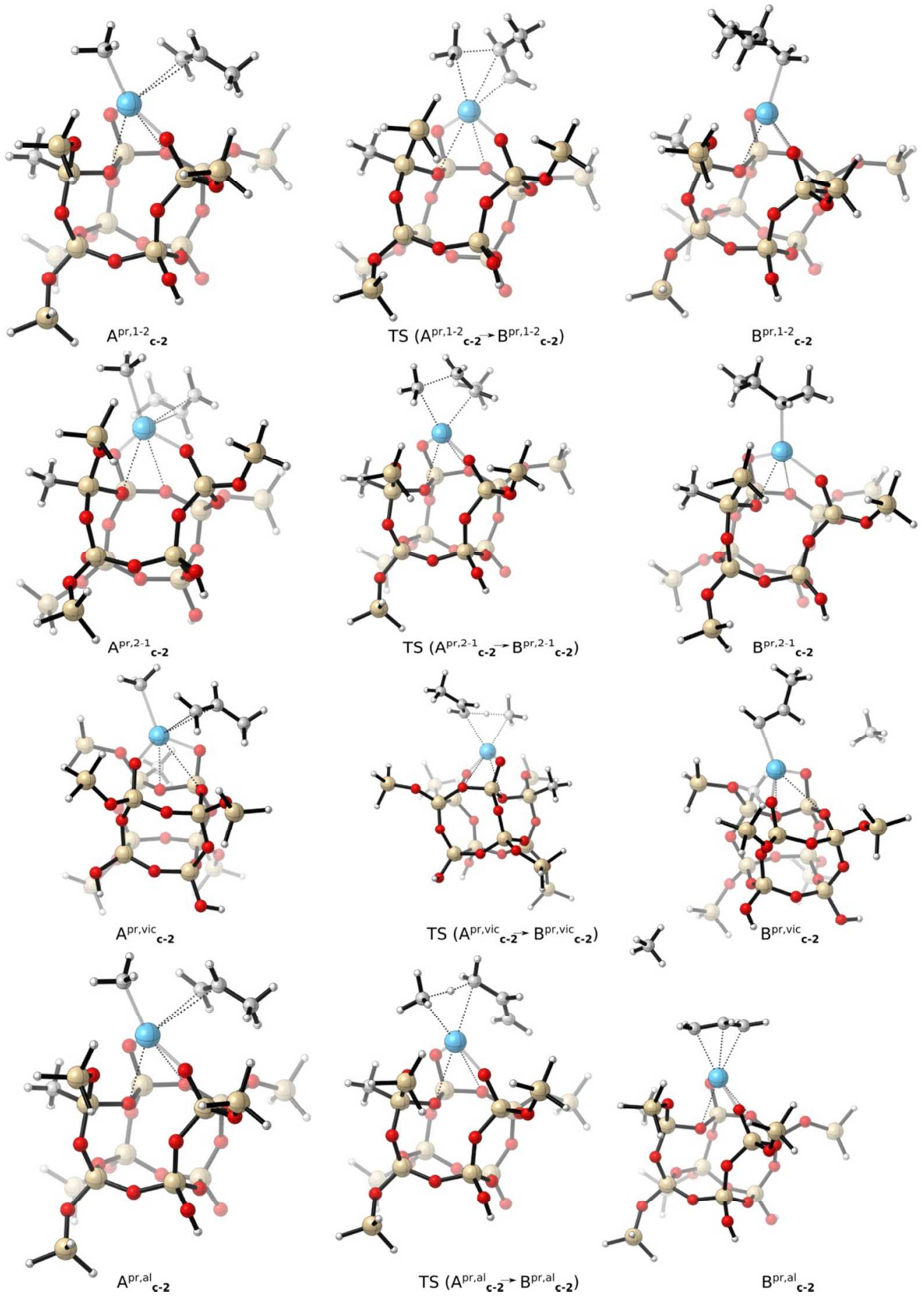

Figure S8. Optimized structures of complexes involved in the initiation step of the propylene 
polymerization mediated by [La]@c-2, [La] refers to fragments $\mathrm{Si}_{13} \mathrm{O}_{20} \mathrm{H}_{17} \mathrm{La}$.
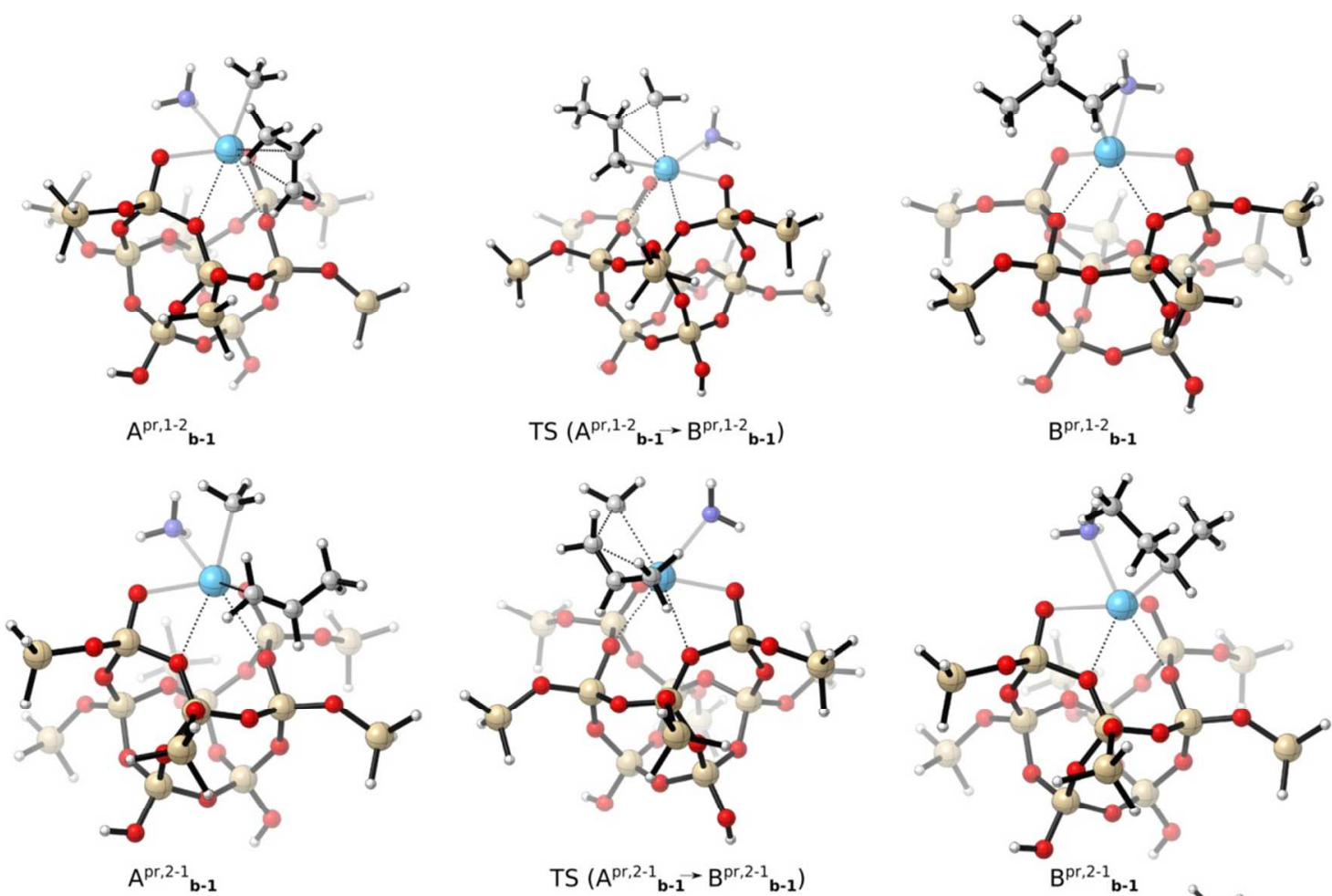

$$
\text { TS }\left(A^{p r, 1-2} \mathbf{b - 1}-B^{p r, 1-2} b-1\right)
$$

$B^{p r, 1-2} b-1$
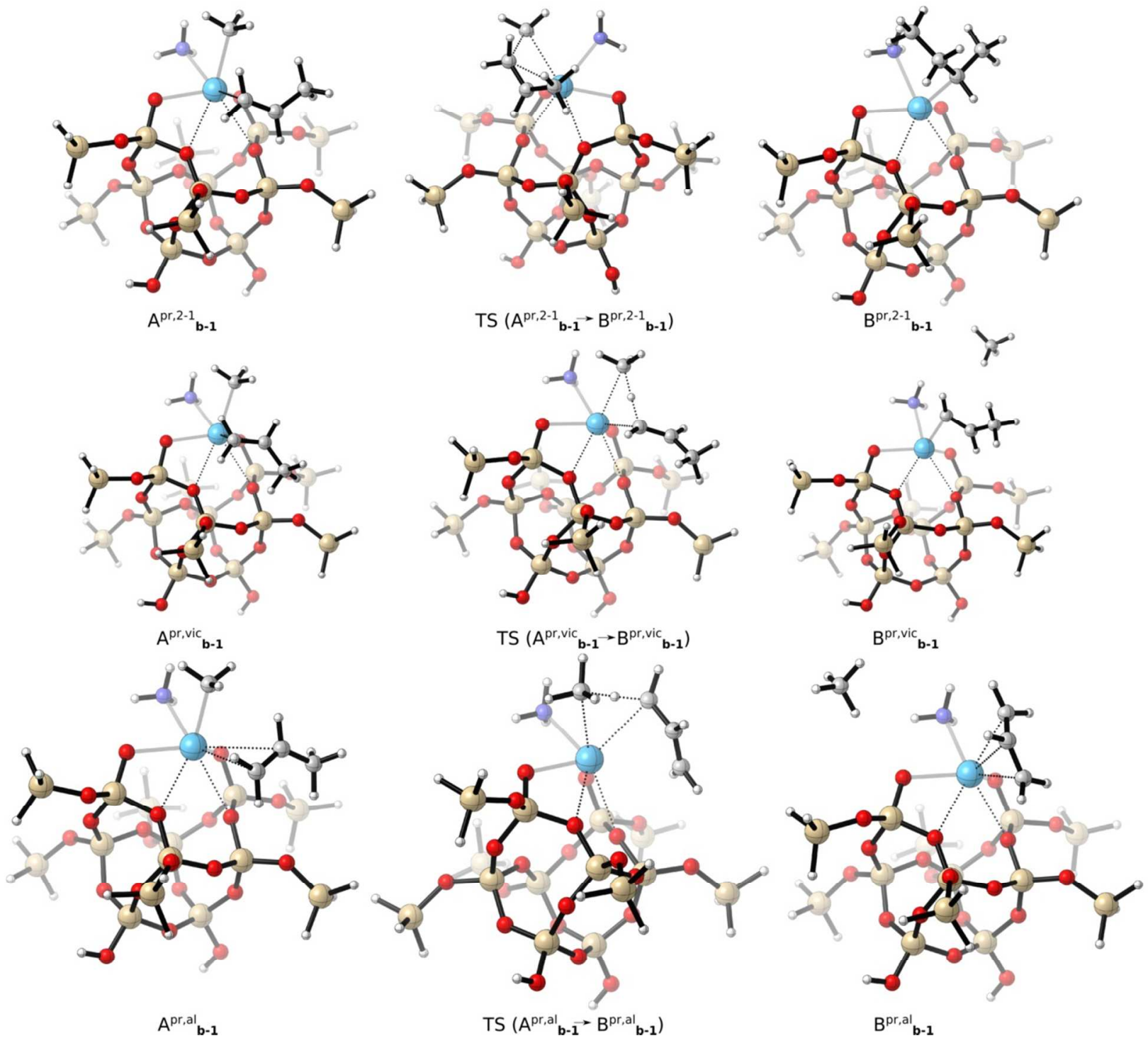

Figure S9. Optimized structures of complexes involved in the initiation step of the propylene 
polymerization mediated by [La]@b-1. [La] refers to fragments $\mathrm{Si}_{14} \mathrm{O}_{21} \mathrm{H}_{20} \mathrm{La}(\mathrm{NH} 3)$.

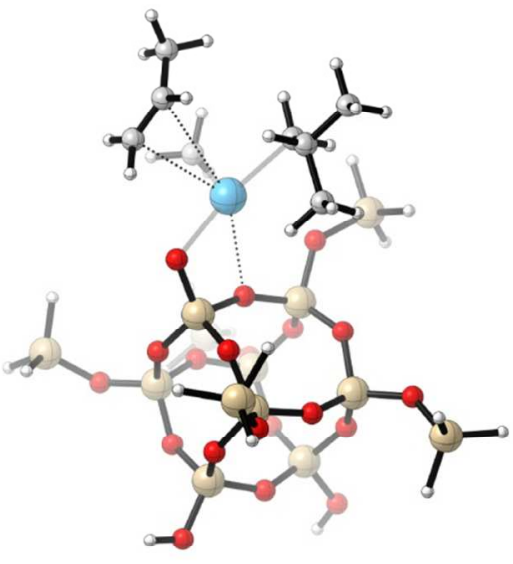

$\mathrm{C}^{\mathrm{pr}, 1-2} \mathrm{c-1}$

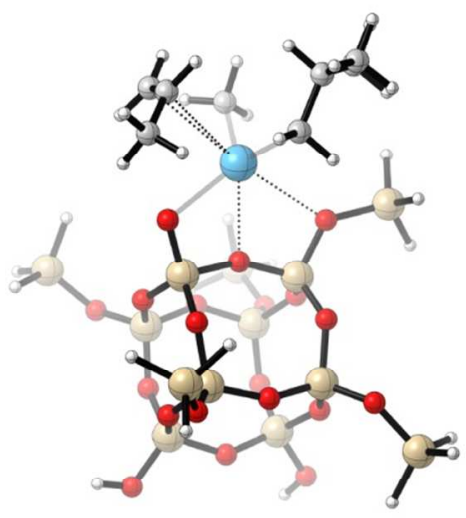

$\mathrm{C}^{\mathrm{pr}, \mathrm{al}}{ }_{\mathrm{c}-1}$

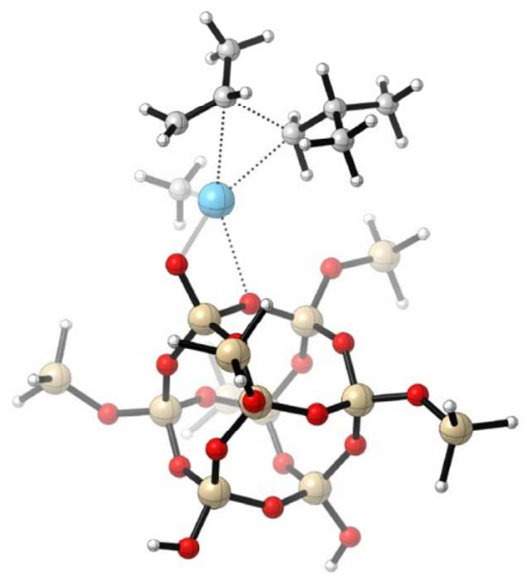

TS $\left(\mathrm{C}^{\mathrm{pr}, 1-2}{ }_{\mathrm{c}-1} \rightarrow \mathrm{D}^{\mathrm{pr}, 1-2}{ }_{\mathrm{c}-1}\right)$

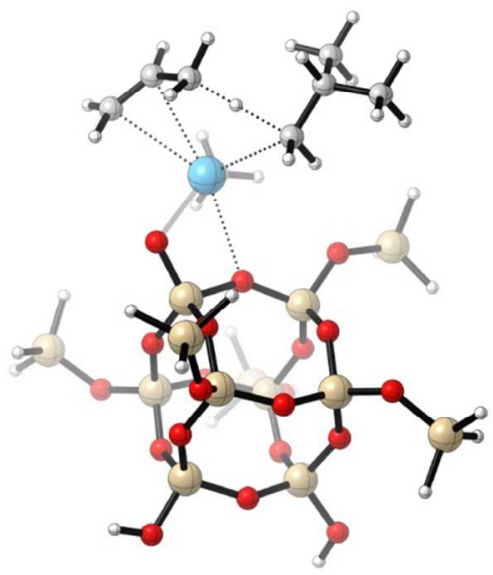

TS $\left(C^{p r, a l}{ }_{c-1} \rightarrow D^{p r, a l}{ }_{c-1}\right)$

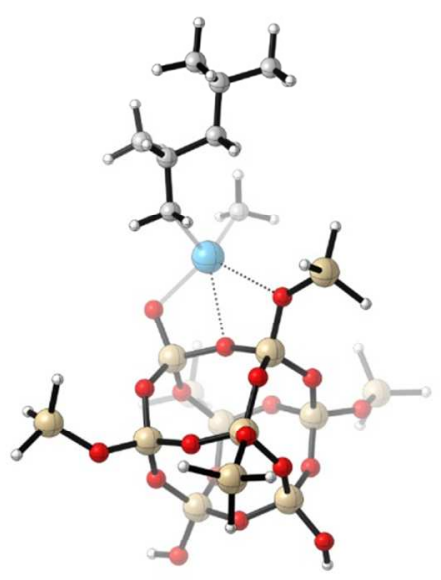

$\mathrm{D}^{\mathrm{pr}, 1-2}$ c-1

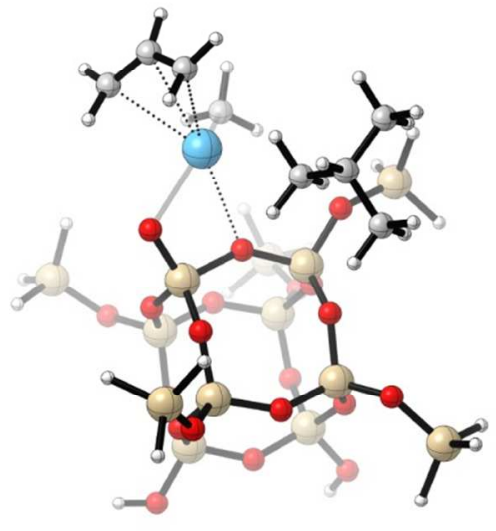

$D^{\text {pr,al }}{ }_{\text {-1 }}$

Figure S10. Optimized structures of complexes involved in the first propagation step of the propylene polymerization mediated by [La]@c-1. [La] refers to fragments $\mathrm{Si}_{13} \mathrm{O}_{20} \mathrm{H}_{17} \mathrm{La}$. 

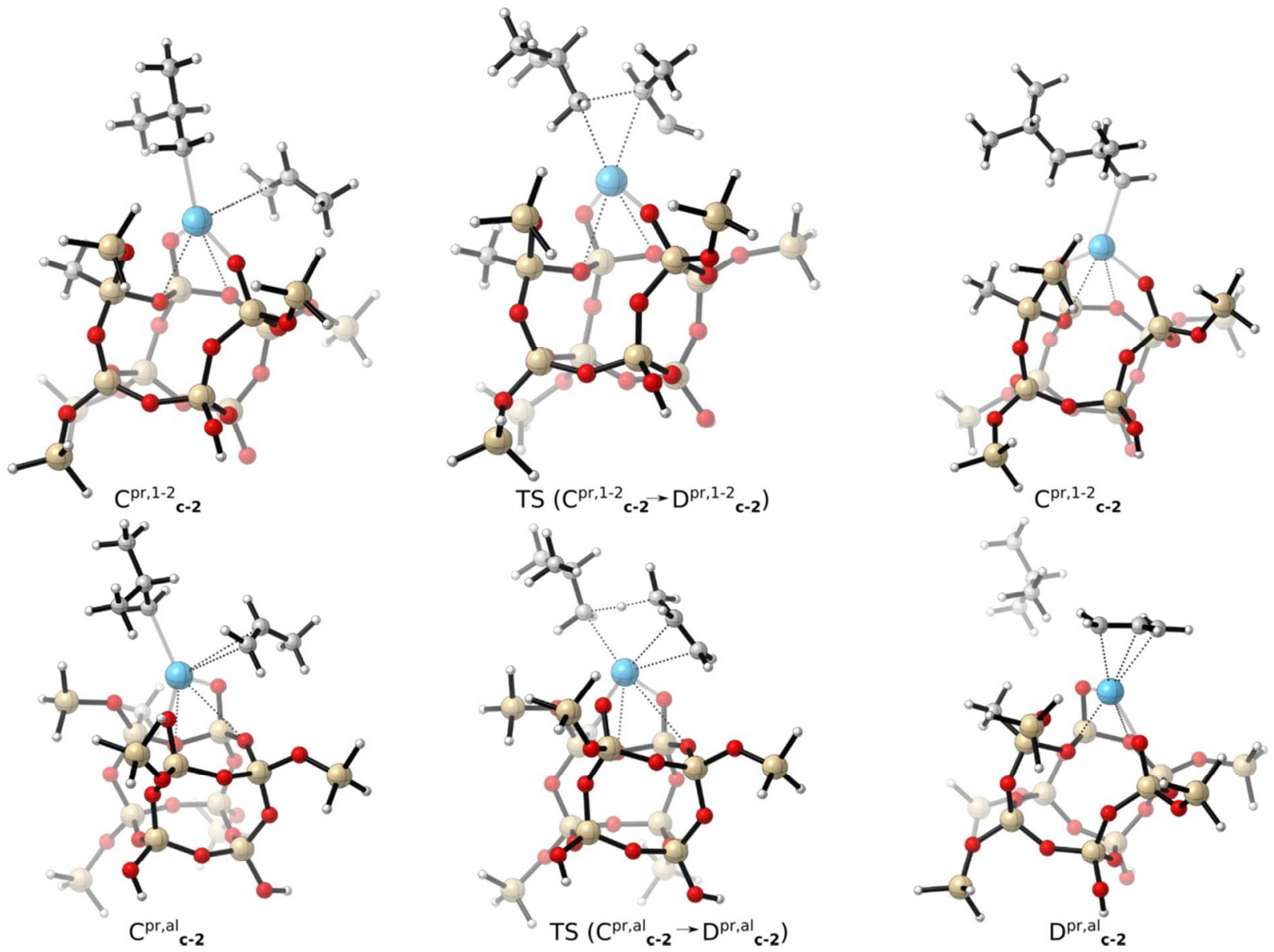

Figure S11. Optimized structures of complexes involved in the first propagation step of the propylene polymerization mediated by [La]@c-2, [La] refers to fragments . 

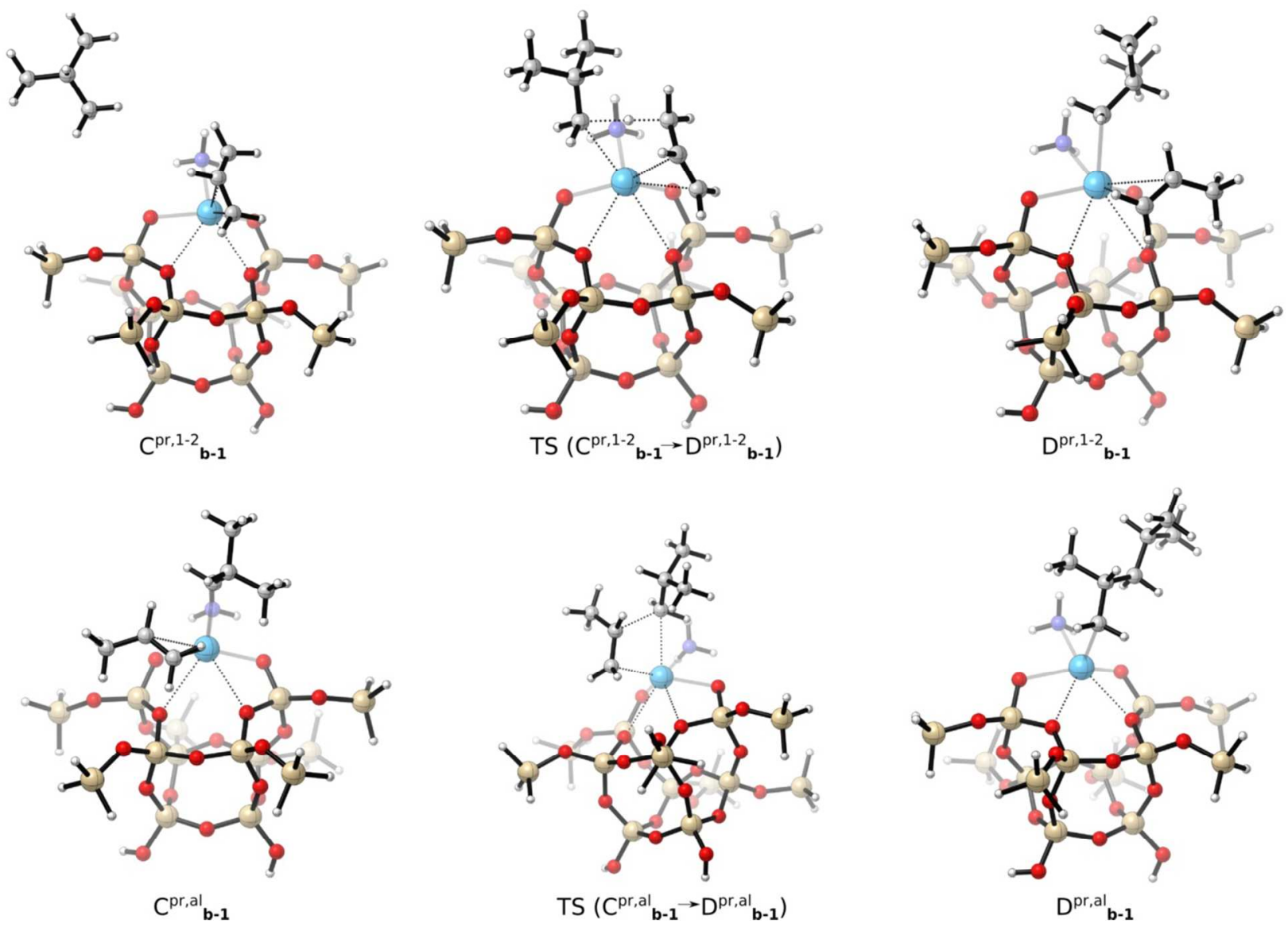

Figure S12. Optimized structures of complexes involved in the first propagation step of the propylene polymerization mediated by [La]@b-1. [La] refers to fragments $\mathrm{Si}_{14} \mathrm{O}_{21} \mathrm{H}_{20} \mathrm{La}(\mathrm{NH} 3)$. 